\title{
In Situ Graphene Liquid Cell Investigation of Metal Ion Modifiers of Calcium Oxalate
}

Lioudmila Sorokina ${ }^{1}$, Abhijit Phakatkar ${ }^{1}$, Reza Shahbazian-Yassar ${ }^{2}$ and Tolou Shokuhfar ${ }^{1}$

${ }^{1}$ University of Illinois at Chicago, United States, ${ }^{2}$ University of Illinois at Chicago, Chicago, Illinois, United States

Calcium oxalate is a salt with ubiquitous presence in nature, including plants and as a part of the nephrolithiasis pathology in humans [1]. Calcium oxalate and calcium oxalate mixed stones constitute approximately $70 \%$ of all urinary stones, thus making it a subject of research interest from both the pathological and materials science perspectives. Precipitation of calcium oxalate is possible due to the excretion pathway of oxalate and calcium, and crystallization is greatest when the two are in a 1:1 ratio. Although supersaturation of calcium oxalate often occurs in vivo, it does not always lead to crystallization due to the naturally occurring inhibitors. Recently, metal ions became of interest as calcium oxalate modifiers due to their ability to affect supersaturation and bind to calcium oxalate in vivo [2].

To elucidate the mechanisms of crystallization, our group has previously identified the classical and nonclassical nucleation pathways using in situ graphene liquid cell (GLC) TEM [3]. Moreover, the addition of citrate, a known inhibitor, led to the formation of a metastable form, calcium oxalate dihydrate, suggesting an increased hydration state of calcium oxalate. The aim of the present study is to discern the effects of metal ions such as iron, nickel, and copper and their ability to modify calcium oxalate crystallization.

Preliminary in situ GLC STEM studies using control calcium oxalate indicate the presence of classical and non-classical mechanisms at atomic level resolution, with evidence of specific non-classical aggregation mechanisms such as coalescence and Ostwald's ripening. Ex situ TEM work provides further supporting evidence of an amorphous to crystalline transition. In addition, both stable and metastable forms of calcium oxalate were observed.

We anticipate that all three metal ions will have a modifying effect on calcium oxalate crystallization. The degree of modification is to be determined.

R. Shahbazian-Yassar and T. Shokuhfar acknowledge the financial support from NSF-DMR 1710049. This work made use of instruments in the Electron Microscopy Core of UIC's Research Resources Center. 


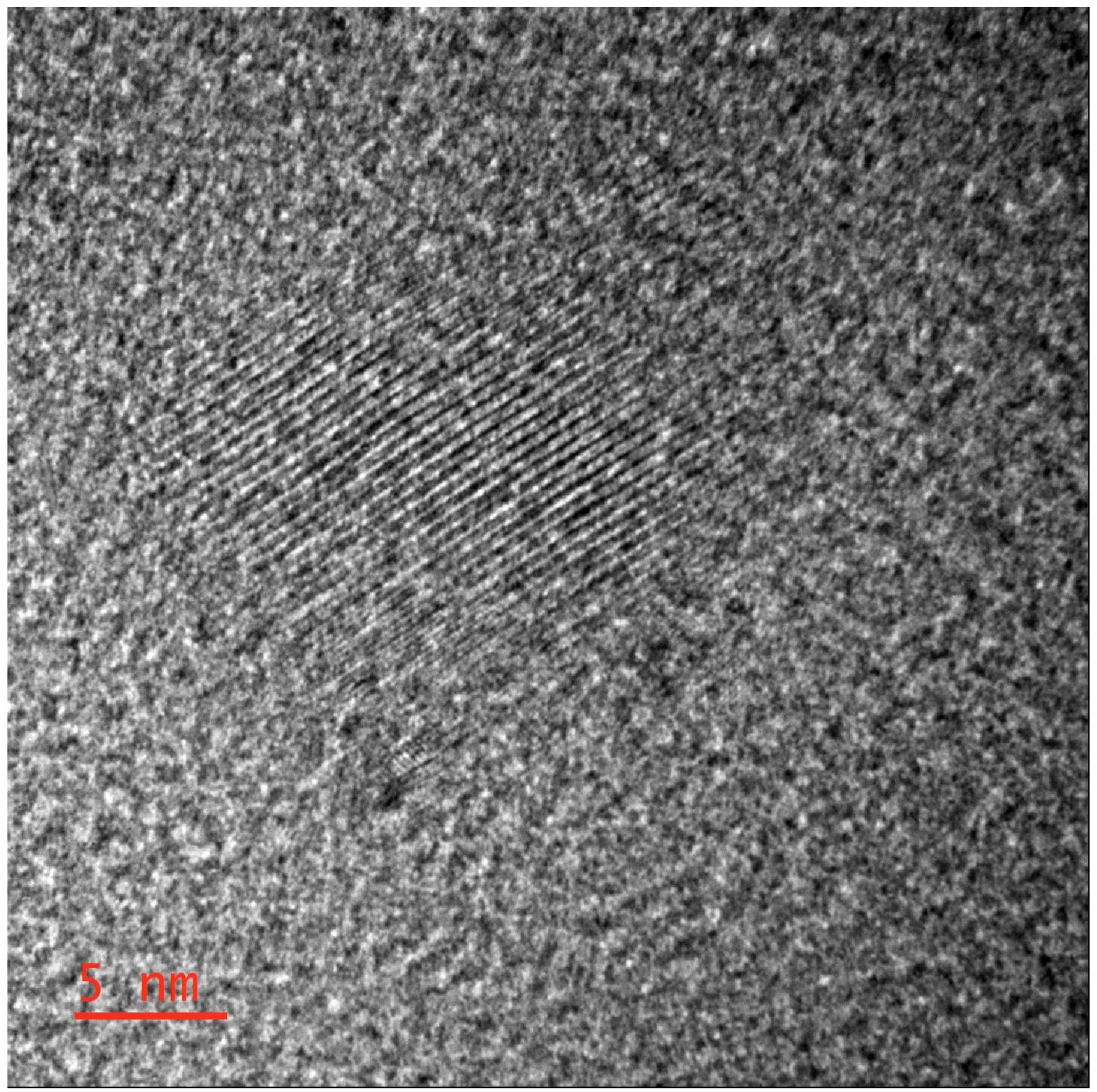

Figure 1. In Situ GLC TEM of Calcium Oxalate - Nucleation 


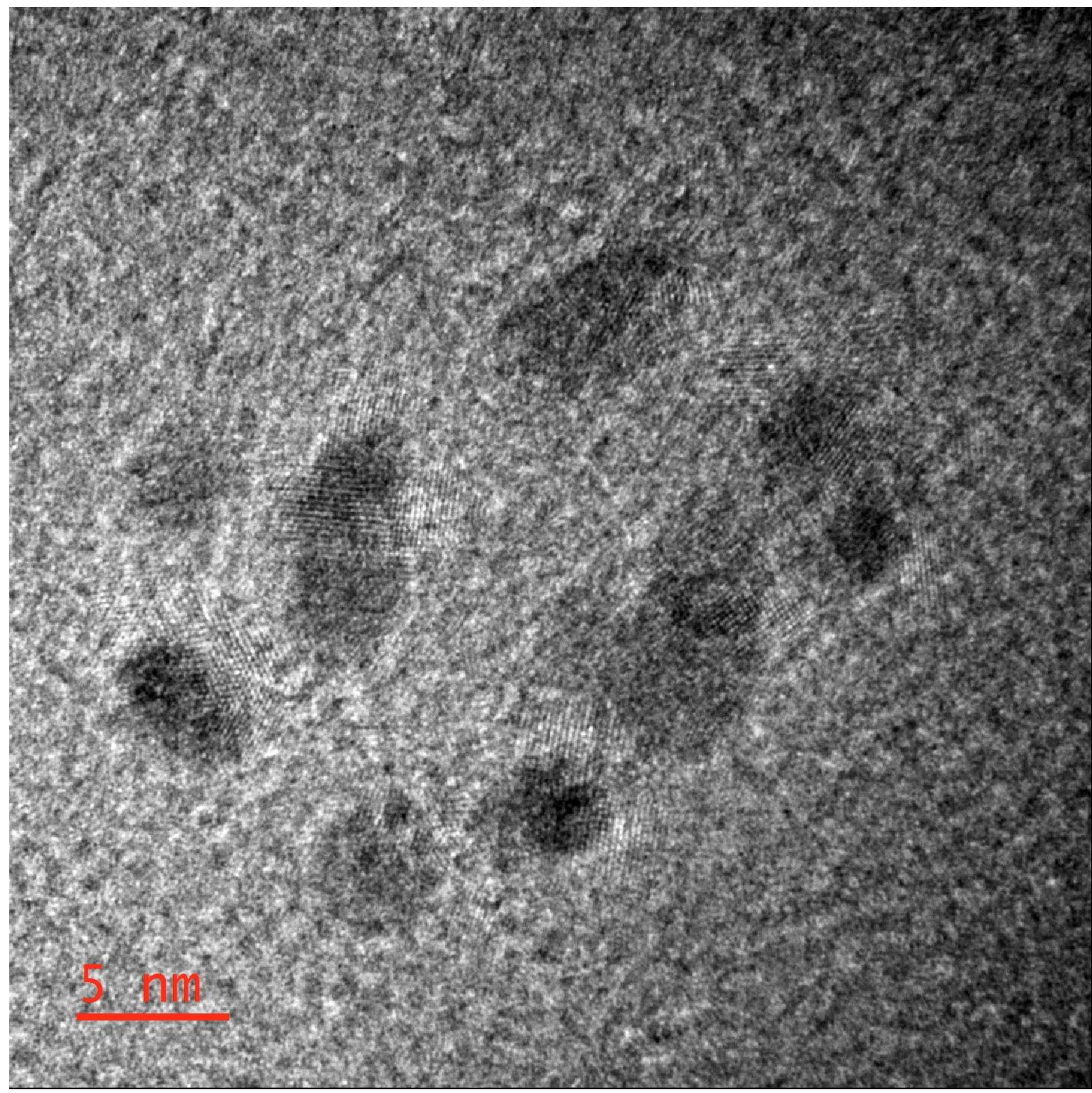

Figure 2. In Situ GLC TEM of Calcium Oxalate - Evidence of Multiple Growth Pathways

\section{References}

[1] Smith, Lynwood H. "The many roles of oxalate in nature." Transactions of the American Clinical and Climatological Association 113 (2002): 1.

[2] Farmanesh, Sahar, Bryan G. Alamani, and Jeffrey D. Rimer. "Identifying alkali metal inhibitors of crystal growth: a selection criterion based on ion pair hydration energy." Chemical Communications 51.73 (2015): 13964-13967.

[3] Banner, David J. et al. "In Situ Liquid Cell TEM Observation of Multiphase Classical and Nonclassical Nucleation of Calcium Oxalate” Advanced Functional Materials (2021) (Accepted) 